\title{
Predictor-Based Robust Control of Uncertain Nonlinear Systems Subject to Input Delay
}

\author{
Serhat Obuz ${ }^{*}$ Enver Tatlicioglu ${ }^{* *}$ Sadettin C. Cekic* \\ Darren M. Dawson* \\ * Department of Electrical \& Computer Engineering, Clemson \\ University, Clemson, SC 29634 USA. (e-mail: \\ [sobuz,scekic,ddarren]@clemson.edu) \\ ** Department of Electrical \& Electronics Engineering, Izmir Institute \\ of Technology, Gulbahce Koyu, Urla, Izmir, 35430 Turkey. (e-mail: \\ envertatlicioglu@iyte.edu.tr)
}

\begin{abstract}
In this paper, a tracking controller is developed for a class of nonlinear systems subject to time delay in the control input, uncertainties in the dynamic model, and additive disturbances. The control development is based on a novel predictor-like method to address the time delay in the control input. Lyapunov based stability analysis is used to prove semi-global asymptotic tracking.
\end{abstract}

Keywords: Robust control, Delay systems, Nonlinear systems, Lyapunov, Tracking control.

\section{INTRODUCTION}

Time delay, also named as time difference of arrival or dead time in different disciplines, is a widely came across phenomenon in dynamical systems in various fields Richard [2003]. Time delay may originate from the dynamics of systems, or may be introduced by the feedback loops, sensors, and communication lines. For example, in telerobotic systems, a slave robot is forced to mimic the motion of a master robot which are usually connected through a communication line, and communication induced time delay may occur and this cause reduction in system performance or even destabilize the closed-loop system Hokayem and Spong [2006]. Mostly to overcome its negative effects (such as instability Datko [1988] and/or reduction in performance), a significant amount of research was devoted to time delay phenomenon, its effects on systems, and identification and control methods. Broad overview on time delay, its effects on systems, and open problems may be found in Richard [2003] and Gu and Niculescu [2003].

Some of the relevant past research focused on analyzing stability of time delay systems. In Schoen [1995], Schoen used Razumikhin theory and Lyapunov-Krasovskii theory to investigate the stability of time delay systems. In Niculescu [2001], Niculescu investigated the effects of time delays on stability of dynamical systems. In Zhong [2006], Zhong investigated robust stability of time delay systems.

A significant amount of research was devoted to designing controllers for systems with input delays. After Smith's pioneering work in Smith [1959] many researchers focused on Smith predictor and modified the predictor for different applications Matausek and Micic [1996], Nortcliffe and Love [2004], Niculescu and Annaswamy [2003], Roca et al. [2009], Majhi and Atherton [2000], Garcia and Albertos
[2008], Manitius and Olbrot [1979], Normey-Rico and Camacho [2009], Kravaris and Wright [1989], Henson and Seborg [1994], Huang and Lewis [2003]. Recently, in Krstic [2009], Krstic considered several systems with input delays and after introducing a transformation, converted the control of systems with input delay problem to boundary control of partial differential equations. Most of the above approaches considered exact knowledge of system dynamics. Some part of the relevant past research focused on developing robust control methods for systems with input delay. The robustness of controllers to uncertainties in input delay Niculescu [2001], Zhong [2006], Krstic [2008], Lozano et al. [2004], model uncertainties Lozano et al. [2004], Roh and Oh [1999], Mirkin and Raskin [2003], Marinescu and Bourles [2000], additive perturbations Normey-Rico and Camacho [2009], Roh and Oh [1999], Kojima et al. [1994], Normey-Rico and Camacho [2002], Mayne et al. [2005], Teel [1998] were demonstrated. Almost all of these robust controllers were proposed for linear systems and/or partial/full knowledge of system dynamics was assumed.

Review of the relevant past literature highlights the fact that fewer results are available for nonlinear systems. In Krstic [2009], Krstic considered nonlinear systems with long actuator delays where exact knowledge of system dynamics was assumed. In Kravaris and Wright [1989] and Henson and Seborg [1994], Smith predictor based linearizing controllers were developed for nonlinear systems, however plant models were assumed to be available. In Huang and Lewis [2003], Huang and Lewis developed a Smith predictor based controller in conjunction with neural networks for a linearized sub-system of a teleoperator system. In Mazenc and Bliman [2006], nonlinear sytems in feedback form with input delay was considered and Lyapunov-Krasovskii functionals were constructed to stabilize the system. Jankovic Jankovic [2006] proposed a 
composite Lyapunov function containing an integral cross term in conjunction with Lyapunov-Krasovskii functionals to stabilize nonlinear cascade systems. In Teel [1998], Teel developed a Razumikhin type theorem that guarantees input-to-state stability for functional differential equations subject to disturbances. Although discussing nonlinear systems, in Kravaris and Wright [1989], Henson and Seborg [1994], Huang and Lewis [2003], Krstic [2009], Teel [1998], Mazenc and Bliman [2006], Jankovic [2006], exact knowledge of system dynamics were assumed when developing controllers. Recently, in Sharma et al. [2011] (and in its preliminary version Sharma et al. [2010]), Sharma et al. proposed two robust controllers for uncertain EulerLagrange systems subject to additive disturbances and input delay, and Lyapunov-Krasovskii functionals were utilized along with a quadratic Lyapunov function to prove ultimate boundedness of the error signals. According to the authors' best knowledge, robust control of uncertain nonlinear systems subject to input delays while guaranteeing an asymptotic result was not addressed in the literature.

In this paper, output tracking control of uncertain nonlinear systems subject to known input delay is considered. The dynamic model is assumed to be uncertain and also subject to additive disturbances. The main contribution of the proposed development is the design of a filtered error signal that is utilized to obtain a delay free openloop error system. This design of the filtered error signal leads to the development of a predictor-based controller that contains a finite integral of the past control inputs. This design of the filtered error signal also allowed us to utilize integral of the sign of the error terms Xian et al. [2004] in the design of the control input. The robustness properties of the integral of the sign of the error terms are utilized in the Lyapunov based stability analysis and semi-global asymptotic convergence of the error signals are guaranteed.

\section{DYNAMIC MODEL}

Consider the following class of nonlinear systems Krstic [2009]

$$
\begin{aligned}
& \dot{x}_{i}=x_{i+1}, i=1, \ldots,(n-1) \\
& \dot{x}_{n}=f(X)+d(t)+u(t-\tau)
\end{aligned}
$$

where $x_{i}(t) \in \mathbb{R}^{m}, i=1, \ldots, n$, are the system states, $X(t)=\left[\begin{array}{llll}x_{1}^{T} & x_{2}^{T} & \cdots & x_{n}^{T}\end{array}\right]^{T} \in \mathbb{R}^{m n}, f(X) \in \mathbb{R}^{m}$ is an uncertain nonlinear function, $d(t) \in \mathbb{R}^{m}$ represents additive disturbances, and $u(t) \in \mathbb{R}^{m}$ is the control input with $u(t-\tau)$ being the delayed control input where $\tau \in \mathbb{R}$ is the known constant positive time delay. The system model in (1) can be rewritten as

$$
x_{1}^{(n)}=f(X)+d(t)+u(t-\tau) .
$$

The system model in (1) is assumed to satisfy the following properties.

Assumption 1. The nonlinear function $f(X)$ is continuously differentiable up to its second order time derivatives (i.e., $f(\cdot) \in \mathcal{C}^{2}$ ).

Assumption 2. The additive disturbance term is assumed to be continuously differentiable and bounded up to its second order time derivatives (i.e., $d(t) \in \mathcal{C}^{2}$ and $d(t)$, $\left.\dot{d}(t), \ddot{d}(t) \in \mathcal{L}_{\infty}\right)$.

\section{CONTROL DEVELOPMENT}

The control design objective is to develop a predictorbased control law that ensures that $x_{1}(t)$ tracks a reference trajectory and that all signals remain bounded within the closed-loop system. In the subsequent development, the nonlinear function $f(\cdot)$ and the additive disturbance term $d(t)$ will be considered as uncertain; thus, will not be utilized in the control design. To achieve the control objectives, the subsequent development is derived based on the assumption that the system state vector $X(t)$ is measurable.

To quantify the control objective, the output tracking error, denoted by $e_{1}(t) \in \mathbb{R}^{m}$, is defined as

$$
e_{1} \triangleq x_{r}-x_{1}
$$

where $x_{r}(t) \in \mathbb{R}^{m}$ is the reference trajectory satisfying following properties

$$
x_{r}(t) \in \mathcal{C}^{n}, x_{r}^{(i)}(t) \in \mathcal{L}_{\infty}, i=0,1, \ldots,(n+2) .
$$

To ease the presentation of the subsequent development, a combination of the reference trajectory and its time derivatives is defined as $X_{r}(t)=\left[\begin{array}{llll}x_{r}^{T} & \dot{x}_{r}^{T} & \cdots & \left(x_{r}^{(n)}\right)^{T}\end{array}\right]^{T} \in$ $\mathbb{R}^{m n}$.

To facilitate the control design, filtered error signals, denoted by $e_{i}(t) \in \mathbb{R}^{m}, i=2,3, \ldots, n$, are defined as follows

$$
\begin{aligned}
& e_{2} \triangleq \dot{e}_{1}+e_{1} \\
& e_{3} \triangleq \dot{e}_{2}+e_{2}+e_{1} \\
& \quad \vdots \\
& e_{n} \triangleq \dot{e}_{n-1}+e_{n-1}+e_{n-2} .
\end{aligned}
$$

A general expression for $e_{i}(t), i=2,3, \ldots, n$ in terms of $e_{1}(t)$ and its time derivatives can be obtained as

$$
e_{i}=\sum_{j=0}^{i-1} a_{i, j} e_{1}^{(j)}
$$

where the known constant coefficients $a_{i, j} \in \mathbb{R}$ are generated via a Fibonacci number series ${ }^{1}$ Xian et al. [2004]. Another filtered error signal, denoted by $r(t) \in \mathbb{R}^{m}$, is defined by

$$
r \triangleq \dot{e}_{n}+\Lambda e_{n}+u(t-\tau)-u(t)
$$

where $\Lambda \in \mathbb{R}^{m \times m}$ is a constant, diagonal, positive definite, gain matrix. It should be noted that, the above definition for $r(t)$ was introduced to obtain a delay-free openloop error system by cancelling the delayed control input (i.e., $u(t-\tau)$ ) in (2) with the same term with negative sign in (9). It should also be noted that, since $\dot{x}_{n}(t)$ is unmeasurable, $r(t)$ is also unmeasurable. To further facilitate the control development, an auxiliary signal, denoted by $e_{u}(t) \in \mathbb{R}^{m}$, is defined as follows

$$
e_{u} \triangleq u(t-\tau)-u(t)
$$

A filtered version of $e_{u}(t)$, denoted by $e_{u f}(t) \in \mathbb{R}^{m}$, is designed as follows ${ }^{2}$

\footnotetext{
1 By definition, the first two Fibonacci numbers are 0 and 1 , and each remaining number is the sum of the previous two Koshy [2001].

2 Throughout the paper, $I_{n}$ and $0_{m \times r}$ will be used to represent an $n \times n$ standard identity matrix and an $m \times r$ zero matrix, respectively.
} 


$$
\dot{e}_{u f}=-\Lambda e_{u f}+e_{u}
$$

where $e_{u f}\left(t_{0}\right)=0_{m \times 1}$. After utilizing (10) and (11), the expression in (9) can be written as follows

$$
r=\dot{e}_{n}+\Lambda e_{n}+\dot{e}_{u f}+\Lambda e_{u f}
$$

and after defining an auxiliary signal, denoted by $\eta(t) \in$ $\mathbb{R}^{m}$, as follows

$$
\eta \triangleq e_{n}+e_{u f}
$$

the following expression is obtained for $r(t)$

$$
r=\dot{\eta}+\Lambda \eta \text {. }
$$

The auxiliary error signals $e_{u f}(t)$ in (11) and $\eta(t)$ in (13) are introduced to put $r(t)$ in an advantageous form that will later be utilized in the stability analysis.

After utilizing (2), $n^{\text {th }}$ order time derivative of (3), (8) (for $e_{n}(t)$ ) along with (9), the following expression can be obtained

$$
r=x_{r}^{(n)}+\sum_{j=0}^{n-2} a_{n j} e_{1}^{(j+1)}+\Lambda e_{n}-f-d-u(t)
$$

where the fact that $a_{n,(n-1)}=1$ was utilized. The time derivative of (15) can be obtained as follows

$$
\dot{r}=S+\tilde{N}-e_{n}-\dot{u}(t)
$$

where $\tilde{N}(t), S(t) \in \mathbb{R}^{m}$ are auxiliary signals defined as

$$
\begin{gathered}
\tilde{N} \triangleq N-N_{r} \\
S \triangleq N_{r}-\dot{d}
\end{gathered}
$$

with $N\left(X, \dot{x}_{n}, t\right), N_{r}\left(X_{r}, x_{r}^{(n+1)}\right) \in \mathbb{R}^{m}$ being auxiliary signals defined as

$$
\begin{aligned}
& N \triangleq x_{r}^{(n+1)}+\sum_{j=0}^{n-2} a_{n j} e_{1}^{(j+2)}+\Lambda \dot{e}_{n}-\dot{f}+e_{n} \\
& \left.N_{r} \triangleq N\right|_{X=X_{r}, \dot{X}=\dot{X}_{r}}=x_{r}^{(n+1)}-\dot{f}\left(X_{r}\right) .
\end{aligned}
$$

Remark 1. Mean Value Theorem Khalil [2002] can be utilized to obtain the following upper bound

$$
\|\tilde{N}(\cdot)\| \leq \rho(\|z\|)\|z\|
$$

where $\rho(\cdot) \in \mathbb{R}$ is a non-negative, globally invertible, non-decreasing function of its argument, $\|\cdot\|$ denotes the standard Euclidean norm, and $z(t) \in \mathbb{R}^{(n+2) m \times 1}$ is the combined error signal defined as

$$
z \triangleq\left[\begin{array}{lllll}
e_{1}^{T} & \cdots & e_{n}^{T} & e_{u}^{T} & r^{T}
\end{array}\right]^{T} .
$$

Remark 2. Note that, since $N_{r}(t)$ and $\dot{N}_{r}(t)$ are functions of the reference trajectory and its time derivatives (which are bounded functions of time), and since from Assumption $2, d(t)$ and its time derivatives are bounded, it can be concluded that $S(t)$ and its time derivatives are bounded functions of time.

Based on the subsequent stability analysis, the control input is designed as follows

$$
\begin{aligned}
u(t)= & \bar{K}\left(e_{n}(t)-e_{n}\left(t_{0}\right)+\Lambda \int_{t_{0}}^{t} e_{n}(\sigma) d \sigma\right) \\
& +\bar{K} \int_{t_{0}}^{t}(u(\theta-\tau)-u(\theta)) d \theta+\Pi(t)
\end{aligned}
$$

where $\bar{K} \in \mathbb{R}^{m \times m}$ is a positive definite, diagonal, constant, control gain matrix defined as $\bar{K} \triangleq K+I_{m}$ with $K \in$ $\mathbb{R}^{m \times m}$ being a positive definite, diagonal, constant, control gain. In (23), $\Pi(t) \in \mathbb{R}^{m}$ is an auxiliary filter signal updated according to the following law

$$
\dot{\Pi}=\beta \operatorname{Sgn}\left(e_{n}+e_{u f}\right)
$$

with $\Pi\left(t_{0}\right)=0_{m \times 1}$ and $\beta \in \mathbb{R}^{m \times m}$ is a positive definite, diagonal, constant control gain matrix. It should be noted that, $u\left(t_{0}\right)=0_{m \times 1}$. The controller $u(t)$ in (23) is a modified linear controller with robust integral of the sign of the error term for uncertainty compensation and predictor-like feedback term for time delay compensation. The controller requires the current and past values of the control input (i.e., $u(t-\theta) \forall \theta \in[0, \tau])$. The time derivative of the control input is found as

$$
\dot{u}(t)=\left(K+I_{m}\right) r+\beta \operatorname{Sgn}(\eta)
$$

where (9), (13) and (24) were utilized. After substituting (25) into (16), the following closed-loop error system is obtained

$$
\dot{r}=S+\tilde{N}-e_{n}-\left(K+I_{m}\right) r-\beta \operatorname{Sgn}(\eta) .
$$

\section{STABILITY ANALYSIS}

Theorem 1. The controller given in (23) and (24) ensures semi-global asymptotic tracking in the sense that

$$
\left\|e_{1}^{(i)}(t)\right\| \rightarrow 0 \text { as } t \rightarrow \infty, i=0, \ldots, n
$$

provided that the elements of $K$ are selected sufficiently large relative to the system initial conditions, and the following sufficient conditions are satisfied

$$
\begin{gathered}
\beta_{i}>\left\|S_{i}(t)\right\|_{\mathcal{L}_{\infty}}+\frac{1}{\Lambda_{i}}\left\|\dot{S}_{i}(t)\right\|_{\mathcal{L}_{\infty}} \\
\Lambda_{\min }-\delta-\frac{1}{2}>0 \\
1-\tau-\frac{\tau}{4 \delta}>0
\end{gathered}
$$

where $\delta \in \mathbb{R}$ is a positive damping constant, $\Lambda_{\min } \in \mathbb{R}$ denotes the minimum eigenvalue of $\Lambda$, and the subscript $i=1, \ldots, m$ denotes the $i$ th element of the vector or the diagonal matrix.

Proof. See Appendix A.

Remark 3. It should be noted that, only the gain condition in (30) depends on the time delay and it is equivalent to the following

$$
\frac{4 \delta}{4 \delta+1}>\tau
$$

and provided that the damping constant is chosen to satisfy $\delta \gg 1$, the controller can compensate for delay values very close to one second.

\section{CONCLUSION}

A novel robust controller was developed for uncertain nonlinear systems subject to constant input delay, uncertainties in the dynamic model and additive disturbances. In the design of the proposed controller, a predictor-based structure was constructed to compensate for the input delay and robust integral of the sign of the error terms were utilized to compensate for the uncertainties in the 
dynamic model. Lyapunov type stability analysis was used to guarantee semi-global asymptotic tracking of a reference trajectory. When compared with the existing studies in the literature, the key contribution of the proposed work is the development of the first-ever controller that addressed robust control of uncertain nonlinear systems with input delay while guaranteeing asymptotic tracking via Lyapunov based stability analysis. Specifically, the closest work to ours is the work of Sharma et al. in Sharma et al. [2011] where two robust controllers were designed for uncertain Euler-Lagrange systems subject to additive disturbances and constant input delay where for one of the controllers the inertia matrix is considered to be known. In the design of the controllers, standard PID/PD controllers were fused with predictor-based terms to guarantee ultimate boundedness of the tracking error. To compare our work with Sharma et al. [2011], in Obuz et al. [2012] an extension to Euler-Lagrange systems is presented, our robust controller is modified to be applicable to uncertain Euler-Lagrange systems when the inertia matrix is known. It is clear that, our controller guarantees asymptotic convergence of the tracking error to zero while in Sharma et al. [2011] ultimate boundedness of the tracking error was obtained. However, in Sharma et al. [2011], there is no restriction on the amount of delay, while, our stability analysis imposed the restriction on the amount of delay to be less than $1 \mathrm{sec}$.

\section{REFERENCES}

R. Datko. Not all feedback stabilized hyperbolic systems are robust with respect to small time delays in their feedbacks. SIAM J. Control \& Optimization, 26(3):697713, 1988.

Z. Dydek, A. Annaswamy, and E. Lavretsky. Adaptive control and the nasa $\mathrm{x}-15-3$ flight revisited. IEEE Control Syst. Mag., 30(3):32-48, 2010.

P. Garcia and P. Albertos. A new dead-time compensator to control stable and integrating processes with long dead-time. Automatica, 44(4):1062-1071, 2008.

K. Gu and S.-I. Niculescu. Survey on recent results in the stability and control of time-delay systems. Tr. of the ASME, 125(2):158-165, 2003.

M. Henson and D. Seborg. Time delay compensation for nonlinear processes. Industrial \& Engineering Chemistry Research, 33(6):1493-1500, 1994.

P. F. Hokayem and M. W. Spong. Bilateral teleoperation: An historical survey. Automatica, 42(12):2035-2057, 2006.

J. Huang and F. Lewis. Neural-network predictive control for nonlinear dynamic systems with time-delay. IEEE Trans. Neural Networks, 14(2):377-389, 2003.

M. Jankovic. Control of cascade systems with time delay - the integral cross-term approach. In Proc. IEEE Int. Conf. Decision and Control, pages 2547-2552, San Diego, CA, USA, 2006.

H. K. Khalil. Nonlinear Systems, 3rd Edition. Prentice Hall, New York, NY, USA, 2002.

A. Kojima, K. Uchida, E. Shimemura, and S. Ishijima. Robust stabilization of a system with delays in control. IEEE Trans. Automat. Contr., 39(8):1694-1698, 1994.

T. Koshy. Fibonacci and Lucas Numbers with Applications. John Wiley and Sons, Inc., New York, NY, USA, 2001.
C. Kravaris and R. Wright. Neural-network predictive control for nonlinear dynamic systems with time-delay. AIChE Journal, 35(9):1535-1542, 1989.

M. Krstic. Lyapunov tools for predictor feedbacks for delay systems: Inverse optimality and robustness to delay mismatch. Automatica, 44(11):2930-2935, 2008.

M. Krstic. Delay Compensation for Nonlinear, Adaptive, and PDE Systems. Birkhauser, Boston, MA, USA, 2009.

R. Lozano, P. Castillo, P. Garcia, and A. Dzul. Robust prediction-based control for unstable delay systems: Application to the yaw control of a mini-helicopter. Automatica, 40(4):603-612, 2004.

S. Majhi and D. Atherton. Obtaining controller parameters for a new Smith predictor using autotuning. Automatica, 36(11):1651-1658, 2000.

A. Z. Manitius and A. W. Olbrot. Finite spectrum assignment for systems with delays. IEEE Trans. Automat. Contr., 24:541-553, 1979.

B. Marinescu and H. Bourles. Robust state-predictive control with separation property: A reduced-state design for control systems with non-equal time delays. Automatica, 36(4):555-562, 2000.

M. Matausek and A. Micic. A modified Smith predictor for controlling a process with an integrator and long deadtime. IEEE Trans. Automat. Contr., 41(8):1199-1203, 1996.

D. Q. Mayne, M. M. Seron, and S. V. Rakovic. Robust model predictive control of constrained linear systems with bounded disturbances. Automatica, 41(2):219-224, 2005.

F. Mazenc and P. Bliman. Backstepping design for timedelay nonlinear systems. IEEE Trans. Automat. Contr., 51(1):149-154, 2006.

L. Mirkin and N. Raskin. Every stabilizing dead-time controller has an observer-predictor-based structure. Automatica, 39(10):1747-1753, 2003.

S.-I. Niculescu. Delay effects on stability: A robust control approach. Springer, Germany, 2001.

S.-I. Niculescu and A.M. Annaswamy. An adaptive Smithcontroller for time-delay systems with relative degree $n^{*} \leq 2$. Systems \& Control Letters, 49(5):347-358, 2003.

J. E. Normey-Rico and E. F. Camacho. A unified approach to design dead-time compensators for stable and integrative processes with dead-time. IEEE Trans. Automat. Contr., 47(2):299-305, 2002.

J. E. Normey-Rico and E. F. Camacho. Unified approach for robust dead-time compensator design. J. Process Control, 19(1):38-47, 2009.

A. Nortcliffe and J. Love. Varying time delay Smith predictor process controller. ISA Transactions, 43(1): 61-71, 2004.

S. Obuz, E. Tatlicioglu, S. C. Cekic, and D. M. Dawson. Predictor-based robust control of uncertain nonlinear systems subject to input delay. Technical Report CU/CRB/4/24/12/1, Clemson University CRB, April 2012. URL http://www.ces.clemson.edu/ece/crb/ publictn/tr.htm

J.-P. Richard. Time-delay systems: an overview of some recent advances and open problems. Automatica, 39(10): 1667-1694, 2003.

L. Roca, J. Luis Guzman, J. E. Normey-Rico, M. Berenguel, and L. Yebra. Robust constrained predictive feedback linearization controller in a solar 
desalination plant collector field. Control Engineering Practice, 17(9):1076-1088, 2009.

Y.-H. Roh and J.-H. Oh. Sliding mode control with delay compensation for uncertain input-delay systems. In Proc. American Control Conf., pages 309-313, San Diego, CA, USA, 1999.

G. M. Schoen. Stability and Stabilization of Time-Delay Systems. Swiss Federal Institute of Technology, Zurich, Switzerland, 1995.

N. Sharma, S. Bhasin, Q. Wang, and W. E. Dixon. Predictor-based control for an uncertain euler-lagrange system with input delay. In Proc. American Control Conf., pages 1422-1427, Baltimore, MD, USA, 2010.

N. Sharma, S. Bhasin, Q. Wang, and W. E. Dixon. Predictor-based control for an uncertain eulerlagrange system with input delay. Automatica, 47(11):2332 $2342,2011$.

O. J. M. Smith. A controller to overcome dead time. ISA Journal, 6:28-33, 1959.

A. Teel. Connections between Razumikhin-type theorems and the ISS nonlinear small gain theorem. IEEE Trans. Automat. Contr., 43(7):960-964, 1998.

B. Xian, D. M. Dawson, M. S. de Queiroz, and J. Chen. A continuous asymptotic tracking control strategy for uncertain nonlinear systems. IEEE Trans. Automat. Contr., 49(7):1206-1211, 2004.

Q.-C. Zhong. Robust control of time-delay systems. Springer, Germany, 2006.

\section{Appendix A. PROOF OF THEOREM 1}

In this appendix, the proof of Theorem 1 is presented.

Proof. Let $V(\cdot) \in \mathbb{R}$ be a Lyapunov function defined as follows

$$
V \triangleq \frac{1}{2} \sum_{i=1}^{n} e_{i}^{T} e_{i}+\frac{1}{2} r^{T} r+Q+P
$$

where $Q(t), P(t) \in \mathbb{R}$ are defined as follows

$$
\begin{aligned}
& Q \triangleq \int_{t-\tau}^{t}\left(\int_{s}^{t}\|\dot{u}(\theta)\|^{2} d \theta\right) d s \\
& P \triangleq \zeta_{b}-\int_{t_{0}}^{t} r^{T}(\theta)[S(\theta)-\beta \operatorname{Sgn}(\eta(\theta))] d \theta
\end{aligned}
$$

where $\zeta_{b} \in \mathbb{R}$ is a positive constant defined as

$$
\zeta_{b} \triangleq \sum_{i=1}^{m} \beta_{i}\left|\eta_{i}\left(t_{0}\right)\right|-\eta^{T}\left(t_{0}\right) S\left(t_{0}\right)
$$

where the subscript $i=1,2, \cdots, n$ denotes the $\mathrm{i}^{\text {th }}$ element of a vector. It should be noted that, the non-negativeness of $P(t)$, which is essential to prove that $V(\cdot)$ is a valid Lyapunov function, is demonstrated in Obuz et al. [2012]. It should also be noted that, based on the definition of $Q(t)$ in (A.2), it is clear that $Q(t) \geq 0$. Furthermore, following upper bound can be obtained for $Q(t)$

$$
Q(t) \leq \tau \sup _{s \in[t-\tau, t]}\left[\int_{s}^{t}\|\dot{u}(\theta)\|^{2} d \theta\right]=\tau \int_{t-\tau}^{t}\|\dot{u}(\theta)\|^{2} d \theta .
$$

The Lyapunov function in (A.1) can be upper and lower bounded as follows

$$
W_{1}(y) \leq V(y, t) \leq W_{2}(y)
$$

where $W_{1}(y), W_{2}(y) \in \mathbb{R}$ are defined as

$$
W_{1}(y) \triangleq \frac{1}{2}\|y\|^{2}, W_{2}(y) \triangleq\|y\|^{2}
$$

with $y(t) \in \mathbb{R}^{[(n+1) m+2] \times 1}$ being defined as

$$
y \triangleq\left[\begin{array}{lllll}
e_{1}^{T} & \cdots & e_{n}^{T} & r^{T} \sqrt{Q} \sqrt{P}
\end{array}\right]^{T} .
$$

Taking the time derivative of the Lyapunov function in (A.1) results in

$$
\dot{V}=\sum_{i=1}^{n} e_{i}^{T} \dot{e}_{i}+r^{T} \dot{r}+\dot{Q}+\dot{P} .
$$

The first term in the above expression can be written as follows

$$
\begin{aligned}
\sum_{i=1}^{n} e_{i}^{T} \dot{e}_{i}= & e_{1}^{T}\left(e_{2}-e_{1}\right)+e_{2}^{T}\left(e_{3}-e_{2}-e_{1}\right) \\
& +\ldots+e_{n-1}^{T}\left(e_{n}-e_{n-1}-e_{n-2}\right) \\
& +e_{n}^{T}\left(r-\Lambda e_{n}-e_{u}\right) \\
= & -\sum_{i=1}^{n-1} e_{i}^{T} e_{i}-e_{n}^{T} \Lambda e_{n} \\
& +e_{n-1}^{T} e_{n}-e_{n}^{T} e_{u}+e_{n}^{T} r
\end{aligned}
$$

where (5)-(7), (11), and (12) were utilized. After substituting the time derivatives of (A.2) and (A.3), and (A.10) into (A.9), and then cancelling common terms results in

$$
\begin{aligned}
\dot{V}= & -\sum_{i=1}^{n-1} e_{i}^{T} e_{i}-e_{n}^{T} \Lambda e_{n}-r^{T} r \\
& +e_{n-1}^{T} e_{n}-e_{n}^{T} e_{u}+r^{T} \tilde{N}-r^{T} K r \\
& +\tau\|\dot{u}(t)\|^{2}-\int_{t-\tau}^{t}\|\dot{u}(\theta)\|^{2} d \theta .
\end{aligned}
$$

After utilizing Young's inequality, the following upper bounds can be obtained

$$
\begin{aligned}
\left|e_{n}^{T} e_{u}\right| & \leq \delta\left\|e_{n}\right\|^{2}+\frac{1}{4 \delta}\left\|e_{u}\right\|^{2} \\
\left|e_{n-1}^{T} e_{n}\right| & \leq \frac{1}{2}\left\|e_{n-1}\right\|^{2}+\frac{1}{2}\left\|e_{n}\right\|^{2} .
\end{aligned}
$$

Note that Cauchy-Schwartz inequality can be utilized to obtain the following upper bound, Obuz et al. [2012]

$$
\left\|e_{u}\right\|^{2} \leq \tau \int_{t-\tau}^{t}\|\dot{u}(\theta)\|^{2} d \theta
$$

and also note that

$$
\|\dot{u}(t)\|^{2} \leq \int_{t-\tau}^{t}\|\dot{u}(\theta)\|^{2} d \theta .
$$

After utilizing (21), (A.12)-(A.15), the right-hand-side of (A.11) can be upper bounded as follows 


$$
\begin{aligned}
\dot{V} \leq & -\sum_{i=1}^{n-2} e_{i}^{T} e_{i}-\left(\Lambda_{\min }-\frac{1}{2}-\delta\right)\left\|e_{n}\right\|^{2}-r^{T} r \\
& -\frac{1}{2}\left\|e_{n-1}\right\|^{2}-\left(1-\tau-\frac{\tau}{4 \delta}\right) \int_{t-\tau}^{t}\|\dot{u}(\theta)\|^{2} d \theta \\
& +\|r\| \rho(\|z\|)\|z\|-K_{\min } r^{T} r
\end{aligned}
$$

where $\Lambda_{\text {min }}, K_{\text {min }} \in \mathbb{R}$ denote the minimum eigenvalues of $\Lambda$ and $K$, respectively. After utilizing (A.14), damping out the $\|r\| \rho(\|z\|)\|z\|$ term with $-K_{\min }\|r\|^{2}$, and then rearranging results in the following expression

$$
\dot{V} \leq-\left[\kappa-\frac{\rho^{2}(\|z\|)}{4 K_{\min }}\right]\|z\|^{2}
$$

where $\kappa \in \mathbb{R}$ is defined as

$$
\kappa \triangleq \min \left\{\frac{1}{2},\left(\Lambda_{\min }-\frac{1}{2}-\delta\right), \frac{1}{\tau}\left(1-\tau-\frac{\tau}{4 \delta}\right)\right\} .
$$

Provided that the control gains are chosen to satisfy $(29)$ and (30), and the elements of $K$ are chosen sufficiently large relative to the initial values, then the right-handside of (A.17) can be upper bounded as

$$
\dot{V} \leq W(z) \leq W(\bar{y})
$$

where $W(\cdot) \in \mathbb{R}$ denotes the following non-positive function

$$
W(z) \triangleq-\gamma\|z\|^{2} \text { and } W(\bar{y}) \triangleq-\gamma\|\bar{y}\|^{2}
$$

where $\gamma \in \mathbb{R}$ is a positive constant and provided that $K_{\text {min }}$ is selected according to the following sufficient condition

$$
K_{\min } \geq \frac{\rho^{2}(\|z\|)}{4 \kappa} \text { or }\|z\| \leq \rho^{-1}\left(\sqrt{4 \kappa K_{\min }}\right) .
$$

In $(\mathrm{A} .20), \bar{y}(t) \in \mathbb{R}^{[(n+1) m] \times 1}$ is defined as

$$
\bar{y} \triangleq\left[\begin{array}{llll}
e_{1}^{T} & \cdots & e_{n}^{T} & r^{T}
\end{array}\right]^{T} .
$$

Based on (A.1)-(A.8) and (A.17)-(A.20) regions $D$ and $S$ can be defined as follows

$$
\begin{aligned}
& \mathcal{D}=\left\{y:\|y\|<\rho^{-1}\left(\sqrt{4 \kappa K_{\min }}\right)\right\} \\
& \mathcal{S}=\left\{y \in \mathcal{D}: W_{2}(y)<\left(\rho^{-1}\left(\sqrt{4 \kappa K_{\min }}\right)\right)^{2}\right\}
\end{aligned}
$$

Note that the region of attraction in (A.24) can be made arbitrarily large to include any initial condition by increasing $K_{\min }$ (i.e., a semi-global stability result). Specifically, (A.7) and (A.24) can be used to calculate the region of attraction as follows

$$
\begin{aligned}
& W_{2}\left(y\left(t_{0}\right)\right)<\left(\rho^{-1}\left(\sqrt{4 \kappa K_{\min }}\right)\right)^{2} \\
& \Longrightarrow\left\|y\left(t_{0}\right)\right\|<\rho^{-1}\left(\sqrt{4 \kappa K_{\min }}\right)
\end{aligned}
$$

which can be rearranged as

$$
K_{\min } \geq \frac{1}{4 \kappa} \rho^{2}\left(\left\|y\left(t_{0}\right)\right\|\right)
$$

where, after utilizing (A.3) and (A.8), the following explicit expression for $\left\|y\left(t_{0}\right)\right\|$ can be derived

$$
\left\|y\left(t_{0}\right)\right\|^{2}=\sum_{i=1}^{n}\left\|e_{i}\left(t_{0}\right)\right\|^{2}+\left\|r\left(t_{0}\right)\right\|^{2}+\zeta_{b} .
$$

From (A.1), (A.19), (A.24)-(A.26), it is clear that $V(\cdot) \in$ $\mathcal{L}_{\infty} \forall y\left(t_{0}\right) \in \mathcal{S}$; hence $\bar{y}(t) \in \mathcal{L}_{\infty} \forall y\left(t_{0}\right) \in \mathcal{S}$. By using (3), (4) and (8), it can be proven that $X(t) \in \mathcal{L}_{\infty}$ $\forall y\left(t_{0}\right) \in \mathcal{S}$, then, it is clear that $f(X) \in \mathcal{L}_{\infty} \forall y\left(t_{0}\right) \in \mathcal{S}$. Since $r(t) \in \mathcal{L}_{\infty} \forall y\left(t_{0}\right) \in \mathcal{S}$, from (14), it is clear that $\eta(t), \dot{\eta}(t) \in \mathcal{L}_{\infty} \forall y\left(t_{0}\right) \in \mathcal{S}$. The expression in (15) can be utilized along with (4) and Assumption 2 to prove that $u(t) \in \mathcal{L}_{\infty} \forall y\left(t_{0}\right) \in \mathcal{S}$. After using these boundedness statements along with (25), it is clear that $\dot{u}(t) \in \mathcal{L}_{\infty}$ $\forall y\left(t_{0}\right) \in \mathcal{S}$. Since $u(t) \in \mathcal{L}_{\infty}$, from (10), it can be concluded that $e_{u}(t) \in \mathcal{L}_{\infty}$; thus, from (11), it is easy to see that $e_{u f}(t), \dot{e}_{u f}(t) \in \mathcal{L}_{\infty} \forall y\left(t_{0}\right) \in \mathcal{S}$. The previous boundedness statements and Remarks 1 and 2 can be used along with (26), to prove that $\dot{r}(t) \in \mathcal{L}_{\infty} \forall y\left(t_{0}\right) \in \mathcal{S}$. The previous boundedness statements can be utilized along with (12) to prove that $\dot{e}_{n}(t) \in \mathcal{L}_{\infty} \forall y\left(t_{0}\right) \in \mathcal{S}$. These boundedness statements can be used along with the time derivative of (A.20) to prove that $\dot{W}(\bar{y}(t)) \in \mathcal{L}_{\infty} \forall y\left(t_{0}\right) \in$ $\mathcal{S}$; hence $W(\bar{y}(t))$ is uniformly continuous. Standard signal chasing algorithms can be used to prove that all remaining signals are bounded. A direct application of Theorem 8.4 in Khalil [2002] can be used to prove that $\|\bar{y}(t)\| \rightarrow 0$ as $t \rightarrow \infty \forall \bar{y}\left(t_{0}\right) \in \mathcal{S}$. Based on the definition of $\bar{y}(t)$, it is easy to show that $\left\|e_{i}(t)\right\|,\|r(t)\| \rightarrow 0$ as $t \rightarrow \infty$ $\forall y\left(t_{0}\right) \in \mathcal{S}, i=1,2, \ldots, n$. By utilizing (8) recursively, it can be proven that $\left\|e_{1}^{(i)}(t)\right\| \rightarrow 0$ as $t \rightarrow \infty, i=1,2, \ldots, n$ $\forall y\left(t_{0}\right) \in \mathcal{S}$; thus, meeting the control design objective.

Remark 4. As explained in Remark 3, from the proof of Theorem 1, there is an upper bound on time delay that can be compensated (i.e., see (31)). This is a direct consequence of the stability analysis. However, as detailed in Dydek et al. [2010], when Lyapunov based methods are utilized, usually, worst case scenarios are considered. For example, the upper bound in (A.15) is very conservative. Given the conservative nature of the Lyapunov based methods, it can be said that, for some applications, delay values that are greater than $1 \mathrm{sec}$ can be compensated as well. 